Keio Journal of Medicine. Vol, 7 ,

Centenary Number, November, 1958

\title{
L'AMNESIE RETROGRADE APRES LA CURE DE SAKEL
}

\author{
KIYOSHI SUZUKI et FUMIO YAMASHITA \\ Clinique neuro-psychiatrique de la Faculté de Médecine de \\ l'Université KEIO \\ KEIICHIRO AKAI et NOBUO SEKI \\ Clinique neuro-psychiatrique de la celle de l'Université NIPPON
}

(Received on July 31, 1958)

Il existe de nombreux rapports sur les accidents et les incidents au cours de la choc-thérapie. Parmi ces documents, ce qui semble extraordinaire, c'est que peu de gens décrivent les effects secondaires que le traitement provoque sur la fonction mentale. Quant au trouble de mémoire consécutif à la convulsinothérapie, on ne compte qu'une dizaine d'articles. Cela serait dû au fait que l'on est toujours trop tenté par les symptòmes psychiques marqués de la période psychotique pour examiner la mémoire en état de rémission.

Mais, en effet, les patients se plaignent très souvent du déficit de mémoire après la choc-thérapie. En 1938, Plattner(1) rapporta, le premier, 7 cas de troubles de mémoire consécutifs à la cure combinée d'Insuline et de Métrazol, dont 4 cas présentaient le syndrome amnésique. Depuis lors, Tooth et Blackurn(2), Ziskind et ses collaborateurs( ${ }^{(3)}$, Sherman, Mergener et Levitin(4) étudièrent quantitativement les effets de la convulsinothérapie sur la fixation et l'évocation de la mémoire. Dans notre pays, Saito(5) observa le même phénomène.

$\mathrm{Au}$ sujet de l'amnésie rétrograde, Schröder(6) rapporta deux observations après la cure combinée d'Insuline et de Cardiazol. Et, Kurosawa(7) observa aussi un schizophrène qui manifestait le syndrome amnésique après le coma prolongé au cours de la cure de Sakel.

Maintenant nous observons un schizophrène qui montre clairement, après. la cure de Sakel sans incident, l'amnésie rétrograde qui s'étend même jusqu'aux deux années précédant le début de la maladie.

\section{OBSERVATION}

..., sexe masculin, né en 1930,26 ans, étudiant.

Urgence de l'Hospitalisation:

flànant auprès de la gare centrale de Tokyo, avec un eiseau à manche de $1 \mathrm{~m}$., il lutta contre des agents de police qui 
l'arrêtèrent au commissariat. Et, il fut interné dans notre hópital des aliénés pour cause d'insanité.

Hérédité, Caractère, Anamnestiques (par l'inquisition objective): Aucune hérédité psychotique dans sa famille. Le troisième fils de ses parents sains qui avaient six enfants. Non attaqué par aucune maladie grave. Elevé dans une famille bourgeoise. Incliné vers la timidité, la tranquillité, la couardise, la viscosité. Il avança progressivement jusqu'au lycée. Après l'échec à l'examen d'entrée d'une université nationale en 1953, il fut assistant dans école secondaire pendant un an. Au printemps de l'année suivante, venu à Tokyo pour préparer l'examen d'entrée, il occupa un emploi. En mars 1955, il réussit à l'examen d'entrée de la Faculté des Lettres d'une université. L'année suivante, il changea d'études en entrant dans la Faculté de Droit. Cette université est bien connue pour sa Faculté de Droit, d'où le changement de ses études.

Début de sa maladie: Un mois après son changement d'études, en avril 1955 , le malade se plaignit d'insomnie, et sa conduite se relâchait. Il fut d'un optimisme excessif et ne réagit que par la gaieté. Il ne se souciait plus de rien et déambulait d'une rue à l'autre dans la nuit. De plus en plus, il manifestait de l'agitation motrice, avec fuite des idées et loquacité, souvent de l'anxiété et de la peur sans objet. Ses paroles devenaient incompréhensibles. A cause de cela, ses amis furent obligés de l'emmener dans son pays natal pour que ses parents s'occupent de lui. Et il passa un mois dans un hôpital où il subit l'électrochoc (E.C.). Se croyant guéri, à la fin de mai, il revint, seul, à Tokyo. Mais, quelque temps après, il se plaignait de céphalée et craignait l'intrigue que l'attitude des chiens provoquaient en lui des suggestions, et que les chiens et les chats se consultaient sur le problème de sa vie. Alors, il décida d'aller consulter un médecin dans une clinique. Le 2 juin 1955, il se leva vers 4 heures du matin et ouvrit toutes les fenètres de son logement, en criant "La bombe atomique va tomber. Sauvez-vous, tous! C'est dangereux, dangereux". De ce moment jusqu'à l'arrestation à la gare, on ne put pas savoir ce qu'il availt fait.

Evolution: Quand le malade entra, il eut l'aspect d'un bloc, avec mutisme obstiné et refusa de se prêter à l'examen. Il se priva d'alimentation. De plus en plus, son agitation devint violente. Souvent, il brisait les fenêtres et donnait des coups de pied à la porte pour essayer de s'enfuir de sa salle.

Après le quatrième E.C., il prononça des paroles menaçantes: "Pourquoi me piques-tu? Veux-tu faire de moi un cochon? Pour ça, en es-tu responsable?", "Je sais bien qu'on veut met tuer avec du poison". Et puis, nous observâmes en lui ces phénomènes: hallucination auditive verbale, idées de persécution, défense contre des accusations, vol de la pensée, barrage et fading.

Nous lui fimes subir l'E.C., 2 fois par semaine, pendant un mois. Au début de juillet, le malade, en qui les symptûmes schizophréniques disparurent, commença à critiquer son passé pathologique: "Une grave maladie mentale m'a attaqué, sans doute. Je me reposerai en prenant un an de congé". Il adressa à son Université une demande de congé écrite de sa propre main. Son état nous sembla en rémission.

A la mi-aout, l'autisme se manifesta de nouveau chez lui, avec un accent affectif plus accusé. Bientôt, chez lui étaient marqués quelques symptômes catatoniques classiques (stéréotypies, maniérisme, négativisme, souvent catalépsie) : c'est le meme état que celui dans lequel il était hospitalisé. Dans le cours de l'E.C. de nouveau, il dit. 
Tableau 1

né Ville $S$

entré école prim. déménage

fini école prim. 1943 entré école second.

1930

transferé au lycee

fini lycée

déménaǵe

échec entrée univ.

assistant au lycée

Sorti du lycée 1953

échec entrée Univ. 1954

arrivé Tokyo

loớe chez T.

entré Faculté

de Lettres

retourné foyer

transferé Faculté de Droit

rentré foyer

revenu Tokyo

flanéà la Gare

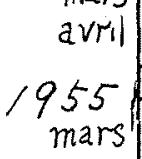

Garcon

Ecolier prim.

Ecolier

second.

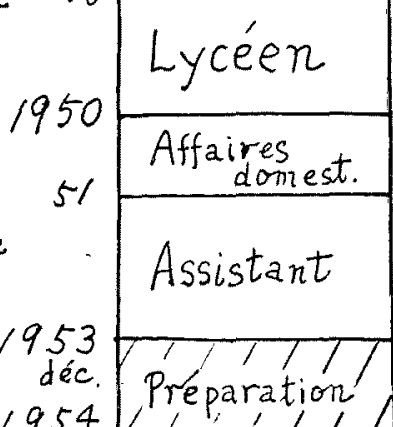

" la période

d'amnésie rétrograde

: le temps pattologique

E.C. : la cure électrochoc

insomnie anxiété flânerie

affectif délire maniérisme abitation hallucination excitation barrage

envoie lettre
à Univ.

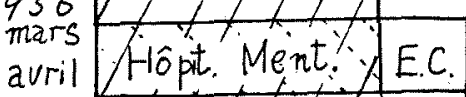

mai

Etudiant

été / duniv. 
"Je suis en Amérique. L'autre moi se trouve près de moi", "Ils, infirmiers et docteurs, me regardent comme ennemi". Nous trouvions en lui la déssorientation, l'idée de persécution, l'agitation motrice forte et l'insomnie.

Donc, nous commençâmes à le traiter par la cure de Sakel.

Modalité technique: D'abord, nous commençâmes par injecter 20 units (u.) d'Insuline qu'on augmente de $10 \mathrm{u}$. chaque jours. La posologie de choc était de $80 \mathrm{u}$. à $90 \mathrm{u}$. Les chocs étaient répétés tous les jours jusqu'à 20 fois. La durée du temps du coma (au commencement 5 minutes, à la fin 30 minutes) était augmentée graduellement de 5 minutes. La posologie d'Insuline compta en total $2385 \mathrm{u}$. Dans cette thërapie, aucun incident ne se produisit, ni l'insulino-résistance vraie, ni la résistance au sommeil, ni le coma prolongé, ni l'état de convulsion.

A partir du 10e coma, nous constatâmes l'affaiblissement de l'excitation motrice et la disparition du symptóme schizophrénique, excépté l'insuffisance de volonté. Mais le malade changea peu à peu d'allégation: "Ce que j'ai fait me semble un reve". A la fin de la cure, il rétablit la susceptibilitié affective et la faculté de modulation en critiquant son passé pathologique et en reprenant l'activité normale.

Après la cure, il se mit à travailler dans le champ de l'hôpital, en état de rémission complète d'après les critères de Sakel et de Müller. Il sortit de l'hôpital avec les meilleurs résultats en fêvrier 1957.

\section{EXAMEN}

Examen physique: Il avait $1.68 \mathrm{~m}$. de taille, $65 \mathrm{~kg}$, de poids. Et, il n'avait aucun signe hématologique, sérologique et urologique. E.C.G. et E.E.G. montraient les tracés normaux. Dans le liquide céphalo-rachidien, tension, albumine, lymphocytes, tout était normal. Les réactions de Pandy, Nonné, Takata-Ara, tout était négatif. Sa maladie n'accompagnait pas le symptôme du système nerveux et des autres organes.

Examen psychologique après la cure: Selon l'échelle de Wechsler Bellevue, le quotient intellectuel verbal montra "115", performance "110", en total "114". Les résultats $d u$ test de Rorschach et $d u$ "thematic apperception test" n'avaient aucun signe curieux. Par les "interviews" narcotiques répétés trois fois (avec l'aide du sodium isoamylethyl barbiturate), nous n'arrivâmes pas à provoquer chez lui des évévements de l'époque où la mémoire lui manquait.

\section{L'AMNESIE RETROGRADE}

\section{1) Début}

Quand la cure fût terminée, le malade se plaigna: "J'ai oublié tous les événements, meme beaucoup de caractères chinois.", et il demanda à une infirmière: “Comment m'a-t-on emmené?". Il écrivit à son père au sujet du motif par lequel il fut venu à Tokyo: "Je regrette bien de ne pas pouvoir me rappeler tout ce qui s'est passé dans ces dernières années". Excepté la pertubation de sa mémoire, il se comporta de façon normale et reconnut exactement le monde extérieur. Il causa longtemps éloquemment avec des malades et 
s'intéressa beaucoup aux événements (le travail, la réunion athlétique).

Klein et $\mathrm{Kral}{ }^{(8)}$ observèrent l'amnésie rétrograde après la contusion cérébrale qui était divisé en trois périodes; (1) celle de la perte totale de conscience, (2) celle du délir, (3) celle de l'amnésie rétrograde. Au Japon, Tanabé(9) a la même opinion. Mais, d'après Kurosawa, le manque de la $2^{\mathrm{e}}$ période caractérise l'amnésie rétrograde après le coma prolongé de la cure de Sakel.

Comme l'observation de Kurosawa le montre, nous ne vîmes pas, chez notre malade, une période qui correspond à la période délirante dans la contusion cérébrale.

Réaction au début: Après la réunion sportive à laquelle le malade participa activement avec gaieté, il se mit à critiquer son passé et à penser à la déficit de sa mémoire, avec les manifestations de l'inquiétude légère que nous n'avons jamais trouvé dans un mois après la cure. Kurosawa trouva que l'amnésie rétrograde de son cas était précédée de l'état d'euphorie durant une semaine après le coma prolongé et que l'amnésie commença par une angoisse très violente. Dans notre cas, cette période précédante fut moins euphorique et plus longue (de 3 semaines), avec une inquiétude moins légère que le cas de Kurosawa. Ces différences dépendraient de celle du coma: "prolongé" ou "non prolongé".

2) Durée

Le malade se plaignit de l'oubli des événements qui se succédaient 2 ans et 11 mois; du décemebre 1953 où il abondonna la fonction d'assistant au lycée pour préparer l'examen d'entrée de l'Université, à l'octobre 1956 où eut lieu la réunion athlétique. Il put se souvenir des faits privés et sociaux dans la période de sa naissance jusqu'à 1953 et du octobre 1956 jusqu'au présent. Ses exposés de sa mémoire sont coïncidentes aux déclarations officielles.

Il n'y a aucune possibilité de croire que sa maladie ait déjà commencé avant l'avril 1956, parce que ses amis et l'hôtesse de sa pension déclarèrent, pour ce qui est de son état de santé mentale, que le malade n'avait pas de signe de neurasthénie, de changement de caractère. En effet, le malade réussit à passer de la Faculté des Lettres à celle de Droit.

Meyer-Gross(10) et Shinkai(11) décrivaient l'amnésie psychotique que leur cas oublia complètement non seulement des symptômes de sa maladie, mais encore des évévements qui s'étaient passé pendant la maladie. Cette amnésie est bien caractérisée par ce qu'elle commence dès le début de la maladie.

Cependant, de ces faits que notre malade était sain avant l'avril 1956 et que son amnésie s'étend jusqu'aux 2 ans précédant le premier signe psychique, et de la définition de Mayer-Gross et Shinkai, nous pouvons conclure que cette amnésie n'est pas l'amnésie psychotique. 


\section{3) Objet}

Kurosawa observa dans son cas l'ile de mémoire dans la période de l'amnésie et l'île d'oubli hors de cette période. Mais Baruk(12) rapporta un cas qui s'était suicidé à cause du déficit de mémoire tellement grave qu'il n'avait pu se souvenir d'aucune connaissance professionnelle après la choc-thérapie. Notre malade oublia des événements privés et sociaux, ainsi que des connaissances qu'il avait acquises à l'université (par exemple le Droit, l'Economie, la Langue allemande). Il ne savait pas prononcer meme des mots allemands très faciles. Au surplus, il oublia même le nom de son université, qu'il avait tant révée pendant si longtemps, à laquelle il avait fait des études plus d'un an. Il lui était impossible de se rappeler le motif par lequel il était venu à Tokyo.

Nous ne constatames pas lile de mémoire dans la période de l'amnésie et

Tableau 2

Le Test des Batteries des Mots

\begin{tabular}{c|cc|ccc}
\hline \multirow{3}{*}{ fois } & \multicolumn{2}{|c|}{ corrélatif } & \multicolumn{3}{c}{ incorrélatif } \\
\cline { 2 - 6 } & $\begin{array}{c}\text { correct } \\
(\%)\end{array}$ & $\begin{array}{c}\text { impossi. } \\
(\%)\end{array}$ & $\begin{array}{c}\text { correct } \\
(\%)\end{array}$ & $\begin{array}{c}\text { erreur } \\
(\%)\end{array}$ & $\begin{array}{c}\text { impossi. } \\
(\%)\end{array}$ \\
\hline \multirow{2}{*}{2} & 100 & 0 & 60 & 30 & 10 \\
3 & 100 & 0 & 60 & 30 & 10 \\
\hline
\end{tabular}

Tableau 3

Le Test d'Ellis

origine reproduction origine reproduction

(1)
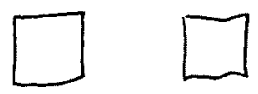

(6)
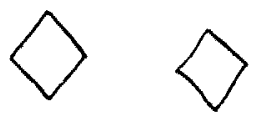

( 7$)$
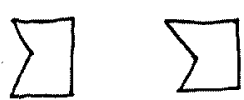

(8)

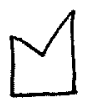

$(q)$

(4)

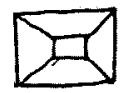

(5)

$(10)$
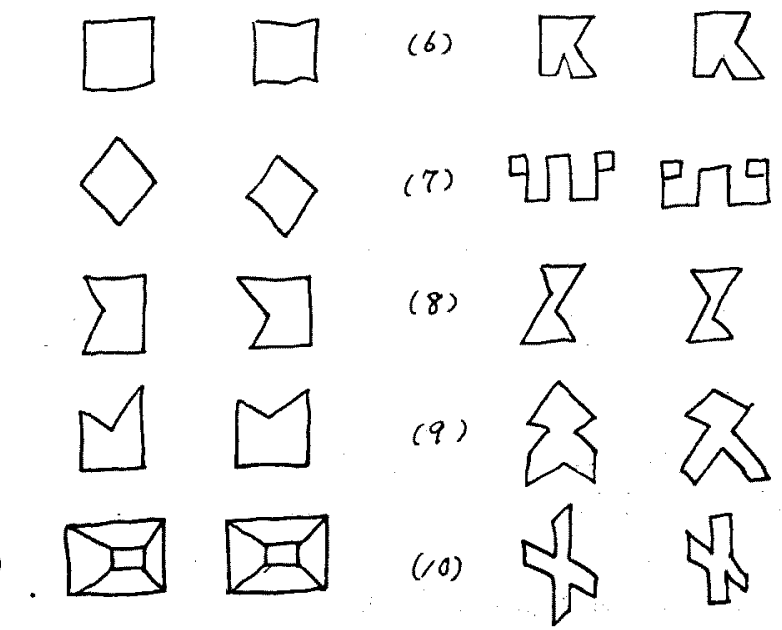
l'ile d'oublie hors de la période de l'amnésie. Donc, notre cas montre l'amnésie: totale.

4) Manque de l'amnésie antérograde

Le malade pouvait acquérir de nouveaux souvenirs. Cliniquement il savait toujours qu'il mangeait et ce qu'il faisait les jours précédants. La mémoire du malade registrait exactement des événements qui succédaient à la réunion sportive dans l'hôpital.

Dans le test d'arrangement d'ustensils et l'examen de Wertheimer, il eut. des meilleurs résultats. Et, en réalité, nous ramenámes le malade deux fois aux lieux où il devait connaitre. La première fois, le malade ne put absolument pas les reconnaítre. La seconde fois, il put se souvenir seulement de cette première. viste.

Cela prouve bien sa bonne reproduction de mémoire.

5) Absence de fabulation et de désorientation.

Nous ne constatâmes pas en lui la désorientation après la fin de la cure. En ce qui concerne l'espace et le temps, l'aliéné s'orientait toujours correctement. Il recommançait à acquérir non seulement la faculté de constater mais encore l'éprouvé global d'existence. Et puis nous ne pûmes pas observer en lui la perturbation de pensée dite par Pick(13) et Grunthal(14) et la paralyse de "couche vitale" dite par Bürger-Prinz ${ }^{(15)}$.

L'examen de temporalisation est à peu près normal.

Tableau 4

La Mesure du Temps présénte

\begin{tabular}{c|c|c|c}
\hline \hline date & $\begin{array}{c}\text { thème } \\
\text { (min.) }\end{array}$ & réponse & (min.) \\
\hline 15 Déc. & 30 & presque & 30 \\
14 Janv. & 20 & à peu près & 20 \\
16 Janv. & 15 & presque & 10 \\
30 Janv. & 40 & presque & 30 \\
3 Féb. & 50 & environ & 60 \\
\hline
\end{tabular}

Tableau 5

La Mesure du Temps percepteur

\begin{tabular}{c|c}
\hline $\begin{array}{c}\text { thème } \\
(\min .)\end{array}$ & $\begin{array}{r}\text { réponse } \\
\text { (min.) }\end{array}$ \\
\hline $\mathbf{5}$ & 7 \\
10 & 15 \\
5 & 6 \\
10 & 10 \\
\hline
\end{tabular}


Tableau 6

La Comparaison de la Durée

\begin{tabular}{|c|c|}
\hline $\begin{array}{l}\text { thème } \\
\text { (min.) }\end{array}$ & réponse \\
\hline $5 \cdots \cdots 5$ & même. \\
\hline $5 \cdots \cdots 3$ & Prem. est longu de $1 \mathrm{~min}$. \\
\hline $3 \cdots \cdots 5$ & Post. est longu de $2 \mathrm{~min}$. \\
\hline $5 \cdots \cdots 5$ & environ même. \\
\hline $10 \cdots \cdots 10$ & environ même. \\
\hline $8 \cdots \cdots 10$ & Post. est longu de $1.5 \mathrm{~min}$. \\
\hline $10 \ldots, 8$ & Prem. est longu de $1 \mathrm{~min}$. \\
\hline
\end{tabular}

Le malade ne remplaçait jamais son manque de souvenir par des récits imaginaires et par des phrases n'ayant aucun rapport avec la réalité des faits. Chez lui, nous n'observàmes pas la fabulation phantastique ou productive.

Takagi(16) rapporta: "l'amnésie rétrograde vient toujous avec le syndrome mental de Korsakow". Mais l'amnésie de notre cas ne montre aucun signe de Korsakow.

6) Tâche de remplir son manque de mémoire

Le malade nous questionnait fréquemment sur les événements dans le passé pathologique et ceux d'avant son hospitalisation. Il envoyait souvent des lettres à ses parents et à ses amis pour se rappeler.

Cette conduite nous permet de nier le syndrome de Korsakow.

\section{DISCUSSION}

Comme ce cas comporte délires, hallucination, hyperkinésies, on peut le mettre dans le cadre de la schizophrénie (catatonique). Pendant le temps pathologique, notre malade montre les symptómes schizophréniques "positifs et négatifs" dits par G. Kloos(17): Il a aussi la discordance, la dissociation et la perte de contact vital avec la réalité (Minkowski) comme le trouble générateur.

On peut nier chez notre malade l'amnésie hystérique bien conne depuis XIXe siècle, parce qu'on ne trouve en lui ni émotion morale vive, ni souffrance. Le malade se contentait bien avant la maladie.

D'autre part, Sataké(18) rapporta un cas de gliome dans la région diencéphalique. Son patient montra d'abord le syndrome hébéphrénique avec l'amnésie rétrograde pendant six mois, et puis, plus tard, il fut atteint de l'hémiparésie gauche et de l'hémianopsie. Mais notre cas manquait et manque encore à présent de syndrome neurologique.

Dans la désorganisation de fonction cérébrale causée par la cure électro- 
simultanée, Nishitani(19) observa quatre degrés: stagnation, démence, amnésie, jugement délabré. $\mathrm{Au}$ processus de la restauration après le traitement, le degré amnésique, qui manque de fabulation, est la plus longue parmi les quatre degrés. Et cette amnésie est réversible. Ce phnénomène ressemble à l'amnésie de notre cas, mais l'amnésie rétrograde de notre malade reste encore incurable.

Selon Ishiguro(20), la quantité de glucose consommée par le cerveau durant le coma d'insuline se diminue sensiblement $(2.1 \mathrm{mg} \%$ ); (en état normal: $9.0 \mathrm{mg} \%$ ).

D'après Shiraki(21) et Otsuka(22), le cerveau mort à cause du coma prolongé d'insuline montre des graves lésions histopathologiques.

Par ces faits, nous assumons que cette amnésie soit provoquée par la désorganisation de fonction cérébrale causée par la cure de Sakel.

\section{RESUME}

Voici les particularités de l'amnésie observée chez un schizophrène qui avait subi la cure de Sakel.

1. Cette amnésie débuta par une légère inquiétude.

2. Elle rétrograduit jusqu'aux deux ans précédant le début de sa maladie. Pendant ce temps, le malade était sain et faisait ses études à l'université.

3. L'objet de cette amnésie était total sans l'ile de mémoire et celle d'oubli.

4. Cette amnésie manquait d'antérogradation.

5. Le malade s'orientait toujours correctement et n'accompagnait pas la fabulation après la cure.

6. La tâche de remplir cette amnésie continuait sérieusement.

La structure de cette amnésie est différente de celle du syndrome de Korsakow, mais elle ressemble à celle de la contusion cérébrale.

Nous pensons donc que cette amnésie fut provoquée par la cure de Sakel.

Les auteurs sont infiniment reconnaissants à Monsieur le Prof. T. Miura de sa direction bienveillante et de son encouragement chaleureux.

Nous exprimons aussi nos sincères remerciements à Dr. Y. Tsujiyama et à Dr. K. Iwasa.

\section{BIBLIOGRAPHIE}

1. Plattner, P.: Amnestisches Syndrom nach Insulin-Cardiazolbehandlung. Z. Neurol. u. Psychiat., 162:728-740, 1938.

2. Tooth, G. and Blackburn, J. M.: Disturbance of memory after convulsion treatment. Lancet, 237:17-20, 1939. 
3. Ziskind, E., Locken, R. and Gengerelli, J. A.: Effect of metrazol on recent learning. Proc. Soc. Exp. Biol., 43:64-65, 1940.

4. Sherman, I., Mergener, J. and Levintin, D.: The effect of convulsive treatment on memory. Amer. J. of Psychiat., 98:401-403, 1941.

5. Saito, M.: Disturbance of memory after convulsion treatment. Encephal. Jap. $2: 271-274,1950$. (in Japanese)

6. Schröder, K.: Retrograde Amnesie nach Krampf-shockbehandlung. Nervenarzt, 13:117-121, 1940.

7. Kurosawa, R.: Uber die amnestischen Syndrome einer Schizophrenen nach dem protrahierten Koma. Psychiat. et Neurol. jap., 45:137-144, 1941. (in Japanese)

8. Klein, R. und Kral, A.: Zur Frage der Pathogenese und Psychopathologie des amnestischen Symptomen-komplexes nach Schädeltraumen. Z. Neur., 149:134-175, 1934.

9. Tanabé, T.: Psychopathology of amnestic syndroms. Dissert. méd. commémo. à Prof. hono. Uématsu:286-303, 1954. (in Japanese)

10. Meyer-Gross, W.: Uber die Stellungsnahme zur abgelaufenen akuten Psychose. Z. Neur., 60:160-212, 1920.

11. Shinkai, Y.: Studies on retropsychotic amnesia. Dissert. méd. commémo. à Prof. hono. Uématsu: 259-267, 1954.

12. Baruk, H.: La thérapeutique par la chorpromazine. Annales méd.-psych. 113e année, T. 1:705-772, 1955.

13. Pick, A.: Beitrag zur Pathologie des Denkverlaufes beim Korsakow. Z. Neur., $28: 344-383,1915$.

14. Grünthal, E.: Zur Kenntnis der Psychopathologie des Korsakowschen Symptomenkomplexes. Mschr. psychiat., 53:89-132, 1923.

15. Bürger-Prinz, H. und Kaila, M.: Uber die Struktur des amnestischen Symptomenkomplexes. Z. Neur., 124:553-559, 1930.

16. Takagi, S.: Uber die psychologische Struktur der retroaktiven Amnesie. Psychiat. et Neurol. jap., 45:539-627, 1941. (in Japanese)

17. Kloos, G.: Psychiatrie und Neurologie. S. 390-416, Rudolph Müller \& Steinicke, 1956.

18. Sataké, R.: Pathologie de la mémoire. P. 42-60, Misuzu-Schobo, Tokyo, 1954. (in Japanese)

19. Nishitani, S.: Clinical Researches on Disturbances in Brain Function following Summation of Electrically induced Convulsion. Psychiat. et Neurol. jap., 54: 1-30, 1952. (in Japanese)

20. Ishiguro, Z.: Clinical Studies on Sugar Consumption by the Brain. Psychiat. et Neurol. jap., 54: 31-54, 1952. (in Japanese)

21. Shiraki, H.: Uber einen Sektionsfall des Protrahierten Insulinkomas Während 89 Tage. Psychiat. et Neurol. Jap., 55:447-463, 1953. (in Japanese)

22. Otsuka, R.: Zur Pathologie des Gehirns eines am protrahierten Insulin-Koma gestorbenen Falls. Psychiat. et Neurol. jap., 56:44-52, 1954. (in Japanese) 